\title{
Buyang Huanwu Decoction promotes neurogenesis via sirtuin 1/autophagy pathway in a cerebral ischemia model
}

\author{
HAN LI $^{1 *}$, DONG PENG ${ }^{1 *}$, SHI-JIE ZHANG ${ }^{2,3^{*}}$, \\ YANG ZHANG $^{1}$, QI WANG ${ }^{4,5}$ and LI GUAN ${ }^{1}$
}

\begin{abstract}
${ }^{1}$ College of Basic Medicine, Guangzhou University of Chinese Medicine, Guangzhou, Guangdong 510006; ${ }^{2}$ Department of Neurology, Guangdong Provincial Hospital of Chinese Medicine; ${ }^{3}$ Department of Neurology, The Second Affiliated Hospital of Guangzhou University of Chinese Medicine, Guangzhou, Guangdong 510120; ${ }^{4}$ Science and Technology Innovation Center, Guangzhou University of Chinese Medicine; ${ }^{5}$ Institute of Clinical Pharmacology, Guangzhou University of Chinese Medicine, Guangzhou, Guangdong 510405, P.R. China
\end{abstract}

Received May 26, 2021; Accepted August 12, 2021

DOI: $10.3892 / \mathrm{mmr} .2021 .12431$

\begin{abstract}
Stroke is one of the main causes of disease-related mortality worldwide. Buyang Huanwu Decoction (BHD) has been used to protect against stroke and stroke-induced disability for several years in China. Studies have shown that BHD can relieve neuronal damage in rats with cerebral ischemia/reperfusion ( $I / R)$ injury. However, the mechanism remains unclear. A middle cerebral artery occlusion and reperfusion (MCAO-R) model was used in the present study. The animals were treated with BHD $(5,10$ and $20 \mathrm{~g} / \mathrm{kg})$ or rapamycin. Infarct size and modified neurological severity score were calculated on day 5 following MCAO-R surgery. Cellular changes around the ischemic penumbra were revealed by hematoxylin and eosin and Nissl staining. The protein expression levels of nestin, brain-derived neurotrophic factor (BDNF), doublecortin on the X chromosome (DCX) and autophagy-related proteins (beclin 1, LC3-II and p62) in the peri-ischemic area of the brain were detected. The results demonstrated that post-surgical treatment with BHD reduced the brain infarct size and improved neurological deficits in MCAO-R rats. BHD protected against MCAO-R-induced neuronal impairment and promoted neurogenesis, increased
\end{abstract}

Correspondence to: Professor Qi Wang, Science and Technology Innovation Center, Guangzhou University of Chinese Medicine, 12 Airport Road, Baiyun, Guangzhou, Guangdong 510405, P.R. China

E-mail:wangqi@gzucm.edu.cn

Professor Li Guan, College of Basic Medicine, Guangzhou University of Chinese Medicine, 232 Outer Ring East Road, University Town, Panyu, Guangzhou, Guangdong 510006, P.R. China

E-mail: guanli@gzucm.edu.cn

${ }^{*}$ Contributed equally

Key words: Buyang Huanwu Decoction, ischemia/reperfusion, neurogenesis, sirtuin 1, autophagy the protein expression of nestin, BDNF and DCX and markedly enhanced autophagy by increasing beclin 1 and LC3-II and decreasing p62. Meanwhile, BHD promoted the expression of sirtuin 1 (SIRT1), an important regulator of autophagy. In conclusion, the present study suggested that post-surgical treatment with BHD could protect rat brains from I/R injury, potentially through the SIRT1/autophagy pathway.

\section{Introduction}

In recent years, stroke has become one of the commonest causes of mortality worldwide $(1,2)$. Stroke occurs when the blood supply to the brain is interrupted or decreased, which prevents the brain tissue from receiving oxygen and nutrients (3). In total, $\sim 85 \%$ of stroke cases are caused by ischemia (4). Stroke can impair neural circuits and function (5). It not only disrupts the infarct area but also the surrounding peri-ischemic areas (6). The lack of blood flow during stroke leads to neural damage, including excitotoxicity, mitochondrial dysfunction, calcium overload, oxidative stress, protein misfolding, inflammatory changes and neuronal apoptosis $(7,8)$. At present, clinical treatments of stroke in the acute phase mainly include thrombolysis, restoration of blood flow in the penumbral area, neurotrophic factor administration to protect neurons and symptomatic treatment. However, a large number of patients with stroke are not suitable for these treatments, due to the narrow time window of the acute phase $(9,10)$. In addition, short-term blood flow recovery usually causes more damage to the neurons $(11,12)$. Stroke is a medical emergency; therefore, early preventive action to reduce brain damage is crucial. However, a limited number of drugs can protect against stroke progression. Thus, identifying alternative therapeutic agents is necessary.

Neurogenesis is the generation of new neurons in the brain, which occurs through the division, maturation and differentiation of neural stem cells (13). Neurogenesis is particularly important in stroke, due to the need for the replacement of cortical neurons destroyed by stroke by new neurons, in order to rebuild neuronal connections (14). Autophagy, the main degradation pathway, is essential for 
maintaining cellular homeostasis (15). Autophagy primarily occurs in peri-ischemic areas during stroke (16). However, the role of autophagy in neuroprotection remains controversial. Certain studies have indicated that the activation of autophagy can promote neuroprotection in stroke $(17,18)$, while others have reported opposite findings (19). Several studies have shown that autophagy serves an important role in neurogenesis while a lack of autophagy-related genes can reduce neurogenesis $(20,21)$.

Proteins from the sirtuins (SIRT) family can mediate autophagy (22). As a type of histone deacetylase, the SIRT family has become an important regulator of metabolism and life span, with SIRT1 being a critical regulator of autophagy (23). SIRT1 can regulate the autophagic pathway under different conditions $(24,25)$. In addition, SIRT1 may serve an important role during stroke progression.

Buyang Huanwu Decoction (BHD) has been used for the treatment of stroke in China for several years (26). Clinical trials have indicated that BHD could ameliorate the outcomes of patients who had suffered a stroke $(5,27,28)$. BHD can protect against cerebral I/R injury by promoting neurogenesis (29-31), inhibiting neural apoptosis and inflammation (32), promoting angiogenesis and improving cerebral circulation (33) in middle cerebral artery occlusion and reperfusion (MCAO-R) rats. Multiple components of BHD could ameliorate the negative effect of stroke (34). Whether the SIRT1/autophagy pathway is involved in the protective effect of BHD against stroke remains to be elucidated.

In the present study, a rat model of MCAO-R was used to determine the neuroprotective effect of BHD in stroke. In addition, it was hypothesized that this effect may be associated with the SIRT1/autophagy pathway.

\section{Materials and methods}

Animals. Male Sprague-Dawley rats $(\mathrm{n}=60$; age, 8 weeks; weight, 270-280 g) were supplied by the Experimental Animal Centre of Guangzhou University of Chinese Medicine (Guangzhou, China). All rats were raised in a specific-pathogen free room under controlled temperature $\left(24 \pm 1^{\circ} \mathrm{C}\right)$ and humidity $(55-70 \%)$ with a 12-h light-dark cycle, and were given free access to food and water. All experimental procedures were carried out according to the guidelines of the Administrative Panel on Laboratory Animal Care of Guangzhou University of Chinese Medicine (Guangzhou, China). After 1 week of adaptive housing and feeding, animals were randomly divided into six groups ( $\mathrm{n}=10$ each group): i) Sham surgery (control); ii) MCAO-R surgery (model); iii) $\mathrm{MCAO}-\mathrm{R}+$ rapamycin (Rapa); iv) $\mathrm{MCAO}-\mathrm{R}+5 \mathrm{~g} / \mathrm{kg}$ BHD; v) MCAO-R + $10 \mathrm{~g} / \mathrm{kg}$ BHD; and vi) MCAO-R + $20 \mathrm{~g} / \mathrm{kg}$ BHD.

Rat model of MCAO-R. A transient focal cerebral ischemia model (MCAO-R model) was established as previously described $(18,35)$. Briefly, rats were anesthetized with $4 \%$ isoflurane and maintained with $1.5 \%$ isoflurane via an isoflurane vaporizer (RWD Life Science). A midline neck incision was then performed to expose the right common carotid artery, external carotid artery (ECA) and internal carotid artery (ICA). A 4-0 silicone rubber-coated nylon monofilament (cat. no. MSRC43B280PK100; RWD Life Science) was inserted into the ECA and then gently advanced into the ICA, 18-19 mm from the carotid bifurcation, to occlude the beginning of the MCA. After $2 \mathrm{~h}$ of occlusion, the monofilament was gently removed to restore blood flow. Throughout the surgery, the body temperature of all rats was maintained at $\sim 37^{\circ} \mathrm{C}$. Rats were anaesthetized by $1 \%$ sodium pentobarbital $(40 \mathrm{mg} / \mathrm{kg}$ ) and euthanized by cervical dislocation on day 5 following surgery.

Preparation of $B H D$. BHD was prepared according to previously described and the quality control was also achieved (Fig. S1) (36). Briefly, the powdered sample of BHD (143 g) was mixed with Radix astragali, Radix angelicae sinensis, Radix paeoniae rubra, Rhizoma ligustici chuanxiong, Flos carthami, Semen persicae and Lumbricus at a 120:6:5:3:3:3:3 ratio. All ingredients were purchased from Guangzhou Zhixin Chinese Herbal Medicine Co. Ltd. and verified by the Department of Pharmacy, Guangzhou University of Chinese Medicine. The decoction was made by boiling the mixture in 10 times the amount of distilled water at $100^{\circ} \mathrm{C}$ for $60 \mathrm{~min}$. The drug solution was removed for use and the residue was boiled once more. The two solutions were combined and concentrated on a rotary evaporator at $\sim 60^{\circ} \mathrm{C}$. The concentrated medicinal solution was vacuum-cooled and dried twice to form a powder and dissolved in distilled water and the final concentration was $2.0 \mathrm{~g} / \mathrm{ml}$ (equivalent to the dry weight of the raw material).

Drug administration. All groups of rats were treated with an intraperitoneal injection of hydroxychloroquine $(20 \mathrm{mg} / \mathrm{kg}$; cat. no. S4430; Selleck Chemicals) 30 min after surgery (37). BHD powder and Rapa (cat. no. S1039; Selleck Chemicals) were dissolved with $0.9 \%$ saline. At the onset of reperfusion (38), the treatment groups, including the MCAO-R + BHD and MCAO-R + Rapa groups, were treated with BHD (5, 10 and $20 \mathrm{~g} / \mathrm{kg}$ ) by gavage and Rapa $(10 \mathrm{mg} / \mathrm{kg})$ via intraperitoneal injection, respectively.

Neurological scores. The modified neurological severity score, which uses different scores to evaluate motor, sensory, reflex and balance functions, was used to assess neurological deficits on day 5 following surgery, as previously described (39-41). The motor test used a six-point scale to assess the movement and walking ability of the rats (muscle state, abnormal motion and tail lifting test). The sensory test used a two-point scale to assess superficial and deep sensations in the rats (vision, touch and proprioception). The reflex test used a four-point scale to assess shallow and deep reflection in rats. The balance functions test used a six-point scale to assess the movement of rats on the balance beam. The neurological function scores ranged between $0-18$ points, and were graded as follows: Mild damage (1-6), moderate damage (7-12) and severe damage (13-18).

Cerebral infarct size measurement. Following euthanasia, rat brains were rapidly sliced using a rat brain matrix (RWD Life Science) to measure the cerebral infarct size. Continuously cut 5 sections of each brain tissue, $2 \mathrm{~mm}$ each, were made 
$(n=4)$. The sections were stained with 2, 3, 5-triphenyltetrazolium hydrochloride (TTC; cat. no. T8877; MilliporeSigma) at $37^{\circ} \mathrm{C}$ for $15 \mathrm{~min}$ (42). Normal tissues stained red and ischemic tissues, white. Image-Pro Plus 6.0 (Media Cybernetics, Inc.) image analysis software was used for image analysis.

Malondialdehyde (MDA), catalase (CAT) and glutathione peroxidase (GSH-PX) expression measurements. The tissue of the ischemic hemisphere of the rats was chosen for the oxidative stress kit testing $(n=3)$. The brain tissues were homogenized with ice-cold saline and centrifuged at $14,000 \mathrm{x} \mathrm{g}$ for $10 \mathrm{~min}$ at $4^{\circ} \mathrm{C}$. The supernatant was then used to detect the levels of CAT (cat. no. A007-1-1; Nanjing Jiancheng Bioengineering Institute), MDA (cat. no. A003-1-2; Nanjing Jiancheng Bioengineering Institute) and GSH-PX (cat. no. A005-1-1; Nanjing Jiancheng Bioengineering Institute), according to the manufacturer's instructions. Absorbance was measured using a microplate reader with the wavelength of 532, 405 and $412 \mathrm{~nm}$.

Hematoxylin and eosin $(H \& E)$ and Nissl staining. The tissue of the ischemic hemisphere of the rats was chosen for H\&E and Nissl staining testing $(n=3)$. Brain paraffin-embedded sections were deparaffinized and rehydrated in xylene and gradient alcohol. The sections were then washed in PBS (Beyotime Institute of Biotechnology) and underwent H\&E (Beyotime Institute of Biotechnology) or Nissl (Nanjing Jiancheng Bioengineering Institute) staining for $10 \mathrm{~min}$ at $37^{\circ} \mathrm{C}$. The sections were then washed with PBS. Images were captured using a light microscope (Leica Microsystems, Inc.). Image-Pro Plus 6.0 (Media Cybernetics, Inc.) software was used for image analysis.

Western blot analysis. The tissue of the ischemic hemisphere of the rats was chosen for western blotting $(n=3)$. Brain tissue was homogenized in ice-cold RIPA lysis buffer (cat. no. P0013B; Beyotime Institute of Biotechnology) and centrifuged at $12,000 \mathrm{xg}$ for $10 \mathrm{~min}$ at $4^{\circ} \mathrm{C}$. The supernatant was then extracted to determine the total protein concentration using a bicinchoninic acid protein assay (cat. no. P0012S; Beyotime Institute of Biotechnology). Next, the appropriate volume of loading buffer (cat. no. BL511B; Biosharp Life Sciences) was added, followed by boiling for $10 \mathrm{~min}$ at $100^{\circ} \mathrm{C}$. Proteins samples (30 $\mu \mathrm{g}$ per well) were separated using 8 , 10 and $12 \%$ SDS-PAGE gels and transferred onto a PVDF (cat. no. ISEQ00010; cat. no. IPVH00010; MilliporeSigma) membrane. The membrane was blocked with $5 \%$ skimmed milk (cat. no. $1172 \mathrm{GR} 500$; BioForxx) at $37^{\circ} \mathrm{C}$ for $1 \mathrm{~h}$. Then, incubated with primary antibodies against SIRT1 (cat.no.ab189494; 1:1,000; Abcam),LC3 (cat.no. 2775; 1:1,000; Cell Signaling Technology, Inc.), beclin 1 (cat. no. 3738, 1:1,000; Cell Signaling Technology, Inc.), p62 (cat. no. 39749; 1:1,000; Cell Signaling Technology, Inc.), doublecortin on the X chromosome (DCX; cat. no. ab18723; 1:1,000; Abcam), $\beta$-actin (cat. no. 58169; 1:1,000; Cell Signaling Technology, Inc.) at $4{ }^{\circ} \mathrm{C}$ overnight and incubated with goat anti-rabbit IgG (cat. no. S0001; 1:3,000; Affinity Biosciences) or goat anti-mouse IgG (S0002; 1:3,000; Affinity Biosciences) at $37^{\circ} \mathrm{C}$ for $1 \mathrm{~h}$. ECL reagent (cat. no. WBKLS0500; MilliporeSigma) was added to the membrane for visualizing the target bands.
Digital images of the blots were visualized using Image Lab 3.0 software (Bio-Rad Laboratories, Inc.).

Immunofluorescence. The tissue of the ischemic hemisphere of the rats was chosen for immunofluorescence testing $(\mathrm{n}=3)$. Rat sections (10 $\mu$ m each) were blocked with 5\% BSA (Beyotime Institute of Biotechnology) and incubated with primary antibodies for nestin (cat. no. 4760; 1:300; Cell Signaling Technology, Inc.), LC3 (cat. no. 2775; 1:300; Cell Signaling Technology, Inc.), brain-derived neurotrophic factor (BDNF; cat. no. ab108319; 1:300; Abcam) and DCX (cat. no. ab18723; 1:300; Abcam) overnight at $4^{\circ} \mathrm{C}$. The slices were incubated with fluorescence-coupled secondary antibody, anti-mouse IgG (cat. no. 4408; 1:1,000; Cell Signaling Technology, Inc.), anti-mouse IgG (cat. no. 4409; 1:1,000; Cell Signaling Technology, Inc.) or anti-rabbit IgG (cat. no. 4412; 1:1,000; Cell Signaling Technology, Inc.) for $2 \mathrm{~h}$ at $37^{\circ} \mathrm{C}$. Following rinsing, sections were incubated with DAPI (cat. no. P0131; Beyotime Institute of Biotechnology). Fluorescence was detected using a laser scanning confocal microscope (Carl Zeiss AG). Image-Pro Plus 6.0 (Media Cybernetics, Inc.) image analysis software was used for image analysis.

Statistical analysis. Statistical analysis was performed using SPSS version 17 (SPSS, Inc.). Data are presented as the mean \pm standard deviation. One-way ANOVA was applied to analyze differences in data for the biochemical parameters among the different groups, followed by Dunnett's post hoc test, and an unpaired Student's t-test was also used to determine statistical differences. $\mathrm{P}<0.05$ was considered to indicate a statistically significant difference.

\section{Results}

BHD ameliorates infarction and reduces neurological scores following $M C A O-R$. The design of the present study is shown in Fig. 1A. First, the infarct volume was measured. TTC staining demonstrated that the infarct volume in the MCAO-R + BHD group was markedly decreased in a dose-dependent manner compared with that in the MCAO-R group (Fig. 1B and C). The neurological scores following MCAO-R in two BHD groups were improved (Fig. 1D), particularly in the $20 \mathrm{~g} / \mathrm{kg}$ BHD group. A dosage of $20 \mathrm{~g} / \mathrm{kg}$ BHD was selected for the next experiments. These data demonstrated that BHD could effectively ameliorate infarction and reduce neurological scores following MCAO-R.

$B H D$ relieves neuronal oxidative stress damage following $M C A O-R$. To determine whether BHD exerted protective effects against oxidative stress, the level of MDA and the activity of CAT and GSH-PX were detected next (Fig. 2). Compared with the sham group, the MDA level in the MCAO-R group was significantly increased and the activity of CAT and GSH-PX was decreased. However, after the oral administration of BHD, the MDA levels in MCAO-R rats were significantly reduced and the activity of CAT and GSH-PX were significantly increased. These data demonstrated that BHD could relieve neuronal oxidative stress damage following MCAO-R. 


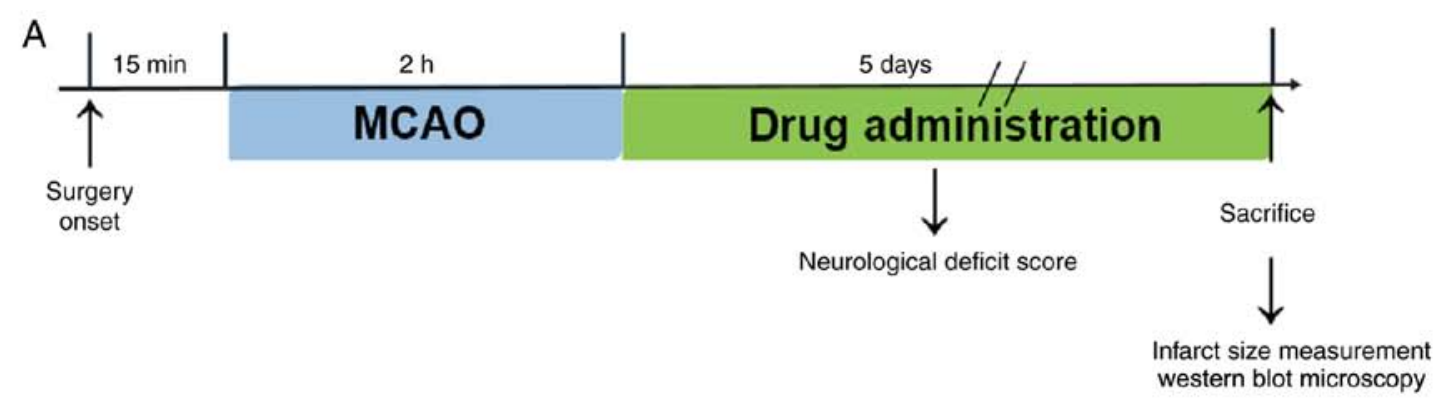

\section{B}
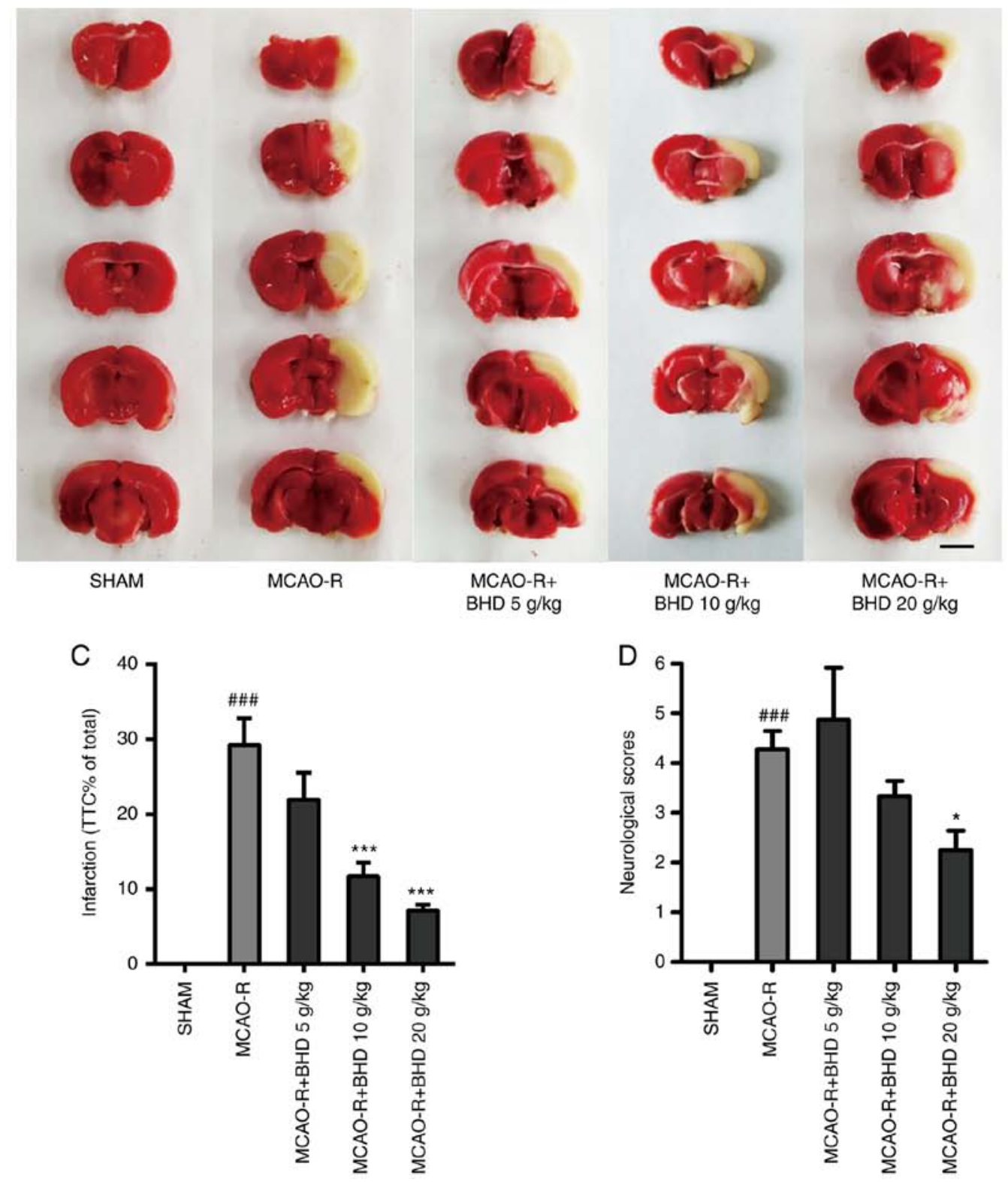

Figure 1. BHD alleviates ischemic brain injury. (A) The schematic protocol of surgery for cerebral ischemia-reperfusion. (B) Rats were subjected to $2 \mathrm{~h}$ of MCAO-R followed by reperfusion. The cerebral infarct volumes were determined by TTC staining. Representative TTC-stained brain slices from each group were shown. Scale bar $=8 \mathrm{~mm}$. (C and D) The infarct volumes and neurological deficit scores of each group were determined. The data were expressed as

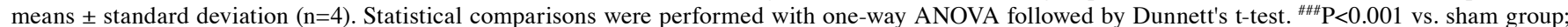
${ }^{*} \mathrm{P}<0.05,{ }^{* * *} \mathrm{P}<0.001$ vs. MCAO-R group. BHD, Buyang Huanwu Decoction; MCAO-R, middle cerebral artery occlusion and reperfusion; TTC, 2, 3, 5-triphenyltetrazolium hydrochloride.

$B H D$ protects against neuronal death following $M C A O-R$. As shown in Fig. 3A and B (H\&E and Nissl staining, respectively), large areas of neuronal necrosis were induced by cerebral
I/R. The MCAO-R group exhibited extensive neuronal death accompanied by the disappearance of cytoplasmic bodies, swelling of cell bodies, nuclear condensation and sparse 

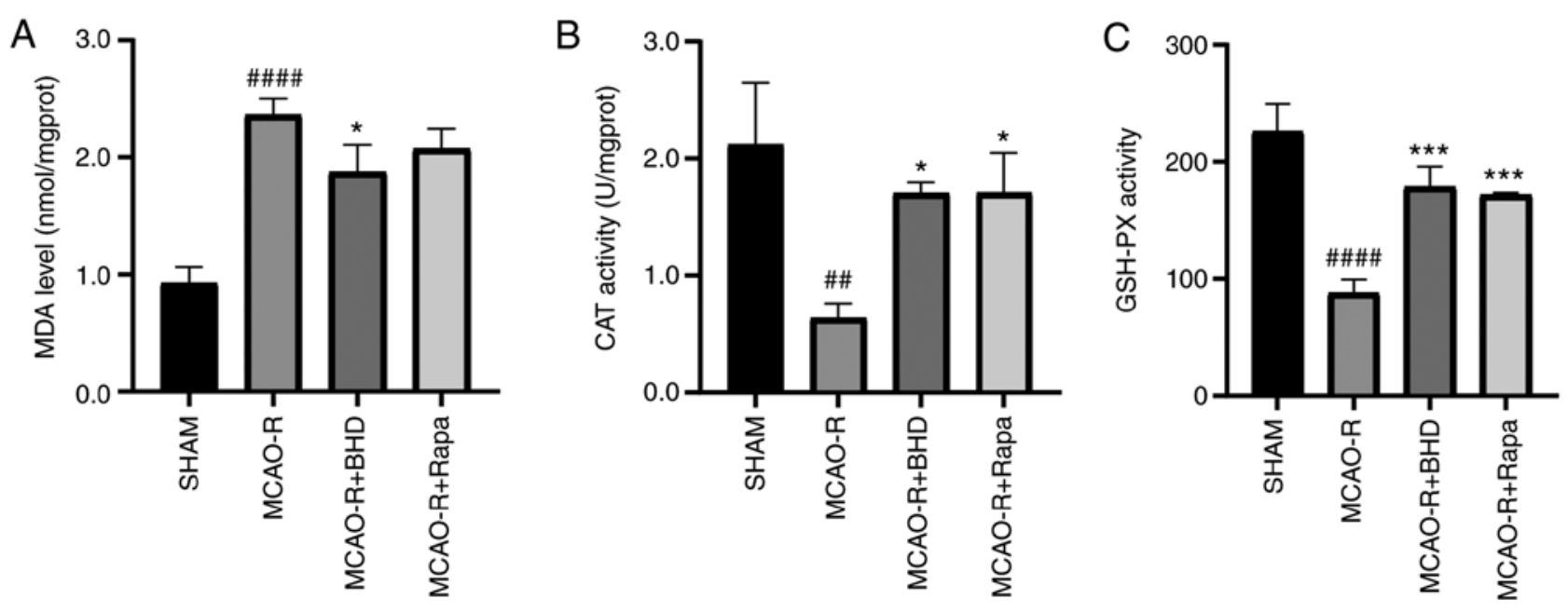

Figure 2. BHD reduces oxidative stress after MCAO-R. The level of (A) MDA and activity of (B) CAT and (C) GSH-PX. Data represent mean \pm standard deviation $(\mathrm{n}=3)$. Statistical comparisons were performed with one-way ANOVA followed by Dunnett's $\mathrm{t}$-test. ${ }^{\# \#} \mathrm{P}<0.01$, ${ }^{\# \# \# \#} \mathrm{P}<0.0001$ vs. sham group; $\mathrm{P}<0.05$, ${ }^{* * *} \mathrm{P}<0.001$ vs. MCAO-R group. BHD, Buyang Huanwu Decoction; MCAO-R, middle cerebral artery occlusion and reperfusion; MDA, malondialdehyde; CAT, catalase; GSH-PX, glutathione peroxidase; Rapa, rapamycin.

Nissl bodies. Conversely, sham group neurons exhibited clear and large cell nuclei and bodies, abundant Nissl bodies and strong staining. Notably, BHD reversed these changes. The expression of BDNF (Fig. 3C and D) was further determined by immunostaining. MCAO-R downregulated BDNF expression. Post-surgical treatment of MCAO-R rats with BHD caused a significant increase in BDNF. Collectively, these data demonstrated that BHD could protect against neuronal death following MCAO-R.

BHD promotes neurogenesis in MCAO-R rats. As shown in Fig. 4, the expression of DCX, a protein expressed by neural precursor cells, was detected using immunostaining and western blot analysis. MCAO-R downregulated DCX. Post-surgical treatment with BHD caused a significant increase in DCX expression in MCAO-R rats. Thus, these results supported that BHD promotes neurogenesis in MCAO-R rats.

BHD activates SIRT1 and autophagy in the cerebral peri-ischemic area of rats following $M C A O-R$. In order to determine whether the SIRT1/autophagy pathway was involved in the protective effect of BHD, SIRT1 and autophagic markers (LC3, p62 and beclin 1) were detected using western blot analysis (Fig. 5A-E). The results demonstrated that the expression of SIRT1 in the MCAO-R and MCAO-R + BHD groups was elevated. SIRT1 expression was significantly increased in the MCAO-R + BHD group, compared with that in the MCAO-R group. The expression of LC3-II and beclin 1 was increased, while that of p62 was slightly decreased in the cerebral peri-ischemic area of rats in the MCAO-R group. Post-surgical treatment with BHD markedly increased autophagy, when compared with the MCAO-R group. Post-surgical treatment with Rapa had a similar effect to that of BHD treatment. In addition, the distribution pattern of nestin and LC3 was elucidated using tissue immunostaining. The expression pattern of LC3 was similar to that observed following western blot analysis (Fig. 6A-C). The expression of nestin, a protein marker for neural stem cells, was also elevated by BHD. Therefore, these data suggested that the neuroprotective effect of BHD might be associated with the SIRT1/autophagy pathway.

\section{Discussion}

In the present study, it was shown that autophagy was activated on day 5 following cerebral I/R in vivo and post-surgical treatment with BHD demonstrated similar trends in regulating autophagy with those of Rapa treatment. Furthermore, the present study found that SIRT1 was upregulated on day 5 following MCAO-R and BHD exacerbated this phenomenon. In addition, BHD treatment increased nestin and DCX expression in MCAO-R rats, suggesting that BHD promoted neurogenesis. Therefore, these results demonstrated that BHD exerts a neuroprotective effect against stroke and promotes neurogenesis, potentially through the activation of the SIRT1/autophagy pathway.

Autophagy, a dynamic process, in which a cell degrades its own cytoplasm through a surrounding lysosome and a bilayer membrane, fluctuates constantly (43). In the central nervous system, moderate autophagy activation may be a manifestation of endogenous neuroprotective mechanisms (44). The formation of autophagosomes, as a process of cell repair and damage limitation following cerebral ischemia, serves a key role in neuronal survival $(45,46)$. In China, BHD has been used for the clinical treatment of stroke for a number of years. BHD protects cerebral ischemia-injured neurons, blood vessels, glial cells and the brain microenvironment through a variety of mechanisms (47-50). In the present study, LC3-II and beclin 1 expression was found to be significantly increased, while p62 expression was found to be decreased, in the peri-ischemic area of rat brains in the MCAO-R + BHD and MCAO-R + Rapa groups, compared with the MCAO-R group. Thus, BHD was shown to activate autophagy in MCAO-R rats, a finding similar to that for Rapa.

Oxidative stress is an important pathological mechanism of stroke (51). When I/R occurs, a large amount of reactive oxygen species (ROS) is produced (52). Mitochondrial 
A

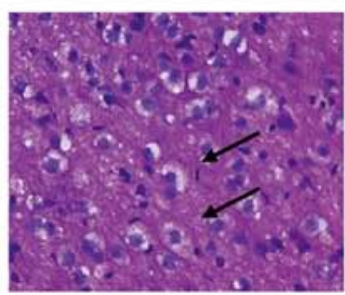

B

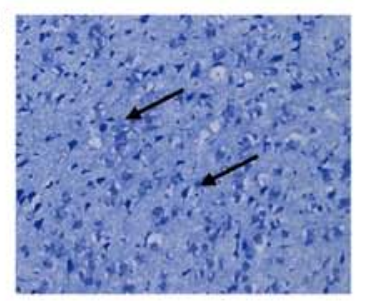

MCAO-R
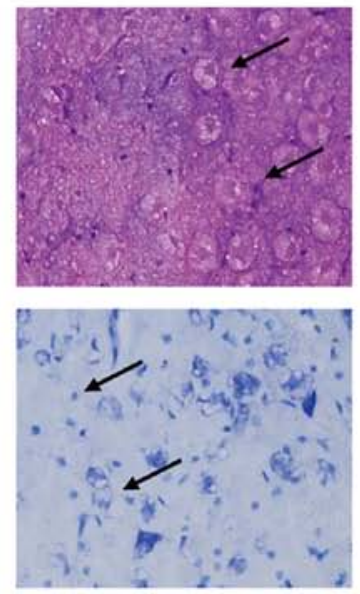

MCAO-R+BHD
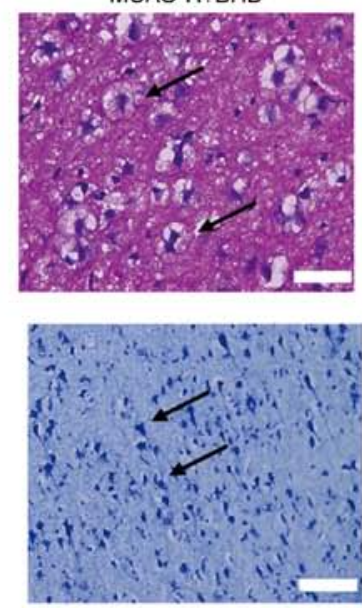

MCAO-R+Rapa

C

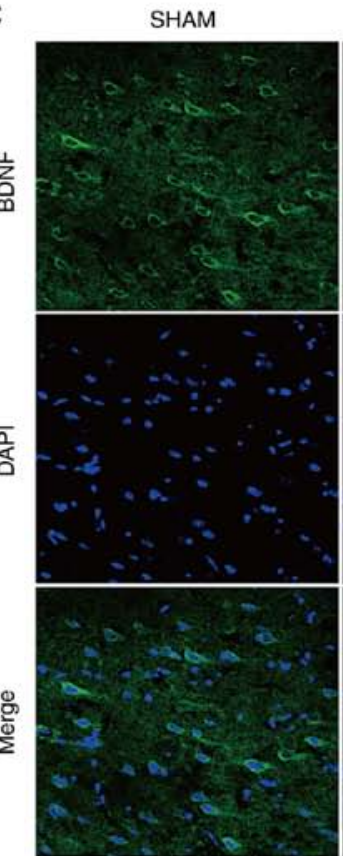

MCAO-R

$M C A O-R+B H D$
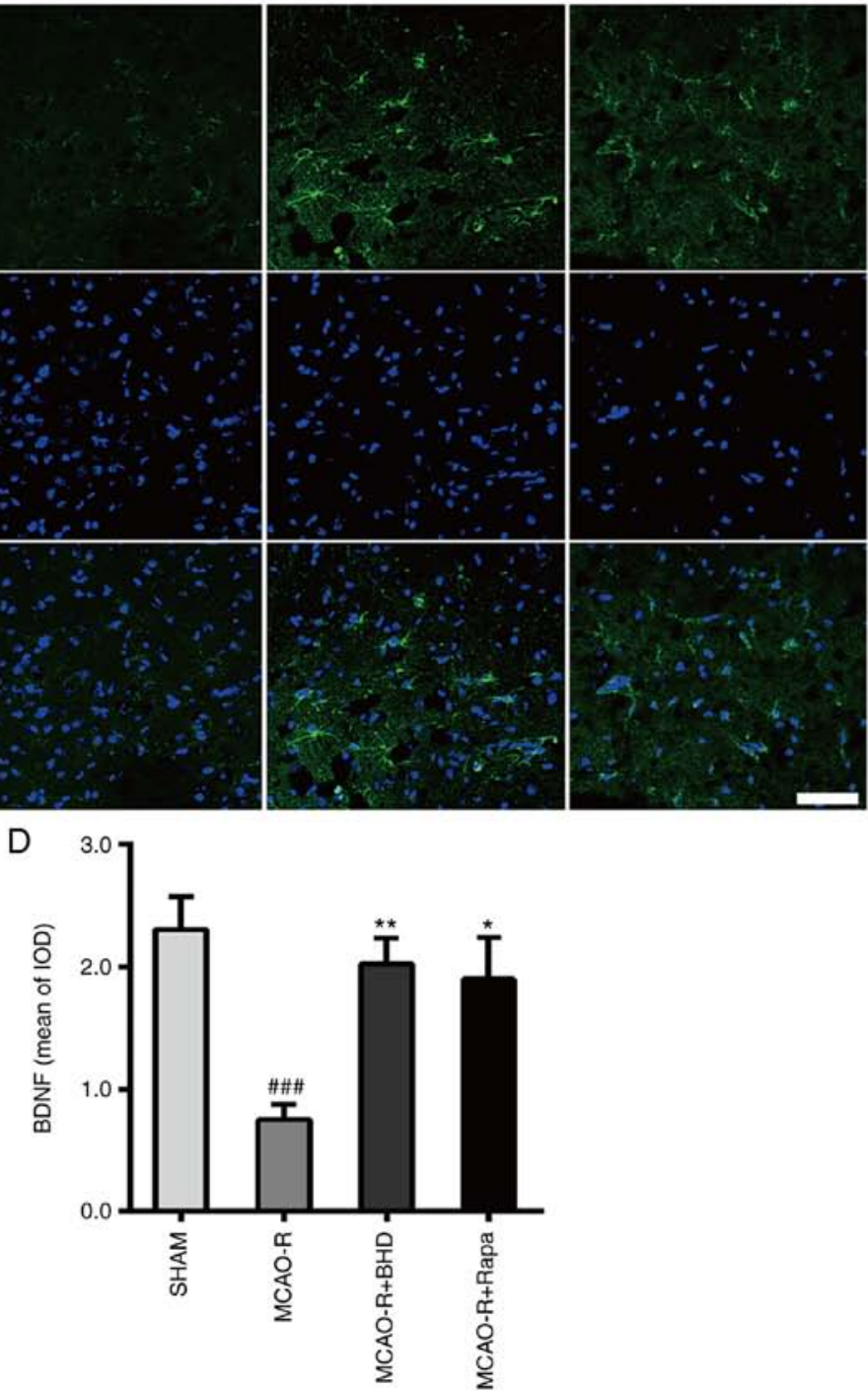

Figure 3. BHD protects against neural death after MCAO-R. (A) Hematoxylin and eosin staining of the ischemic penumbra. Scale bar=50 $\mu \mathrm{m}$. The arrow points to the cytoplasm and nucleus of neurons of sham group, MCAO-R group and MCAO-R + BHD group rats. Sham group neurons demonstrated clear and large cell nucleus and bodies. MCAO-R group neurons demonstrated disappearance of the cytoplasmic bodies, swelling of cell bodies and nuclear condensation. The MCAO-R + BHD group demonstrated neurons clear cell nucleus and bodies. (B) Nissl staining of the ischemic penumbra. Scale bar=50 $\mu \mathrm{m}$. The arrow points to the Nissl body of sham group, MCAO-R group and MCAO-R + BHD group rats. Sham group neurons demonstrated a lot of Nissl bodies and deep staining. MCAO-R group neurons demonstrated sparse Nissl bodies and weak staining. The MCAO-R + BHD group demonstrated more Nissl bodies and deeper staining. (C) Immunofluorescence analysis performed with BDNF antibody (green), nuclei were stained with DAPI (blue). Scale bar=20 $\mu \mathrm{m}$. (D) The bar graph represents the IOD quantification of BDNF. The data are expressed as means \pm standard deviation $(n=3)$. Statistical comparisons were performed with one-way ANOVA followed by Dunnett's post hoc test. ${ }^{\# \# \#} \mathrm{P}<0.001$ vs. sham group; $\mathrm{P}<0.05,{ }^{* *} \mathrm{P}<0.01$ vs. MCAO-R group. BHD, Buyang Huanwu Decoction; MCAO-R, middle cerebral artery occlusion and reperfusion; BDNF, brain-derived neurotrophic factor; Rapa, rapamycin; IOD, integrated optical density. 

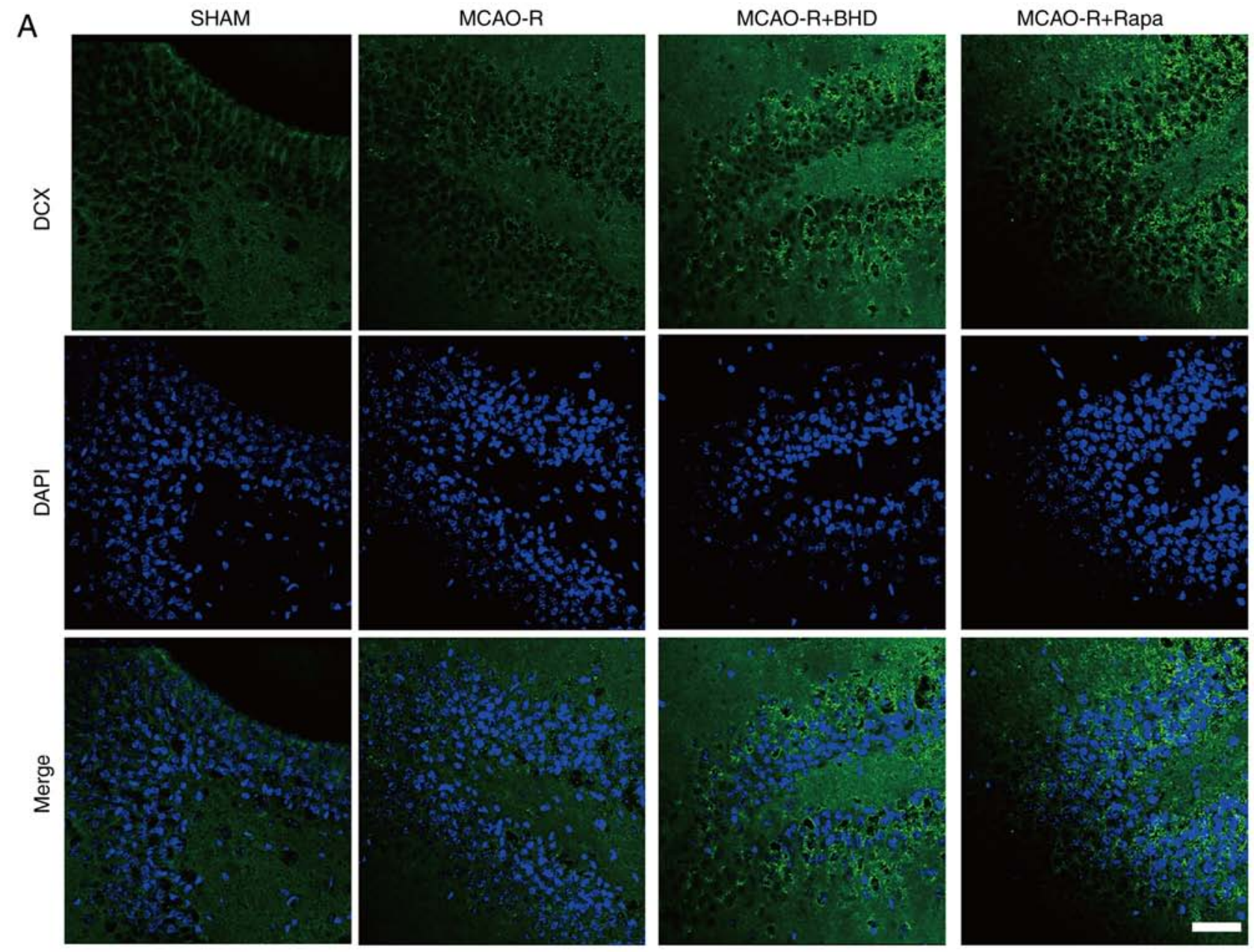

B
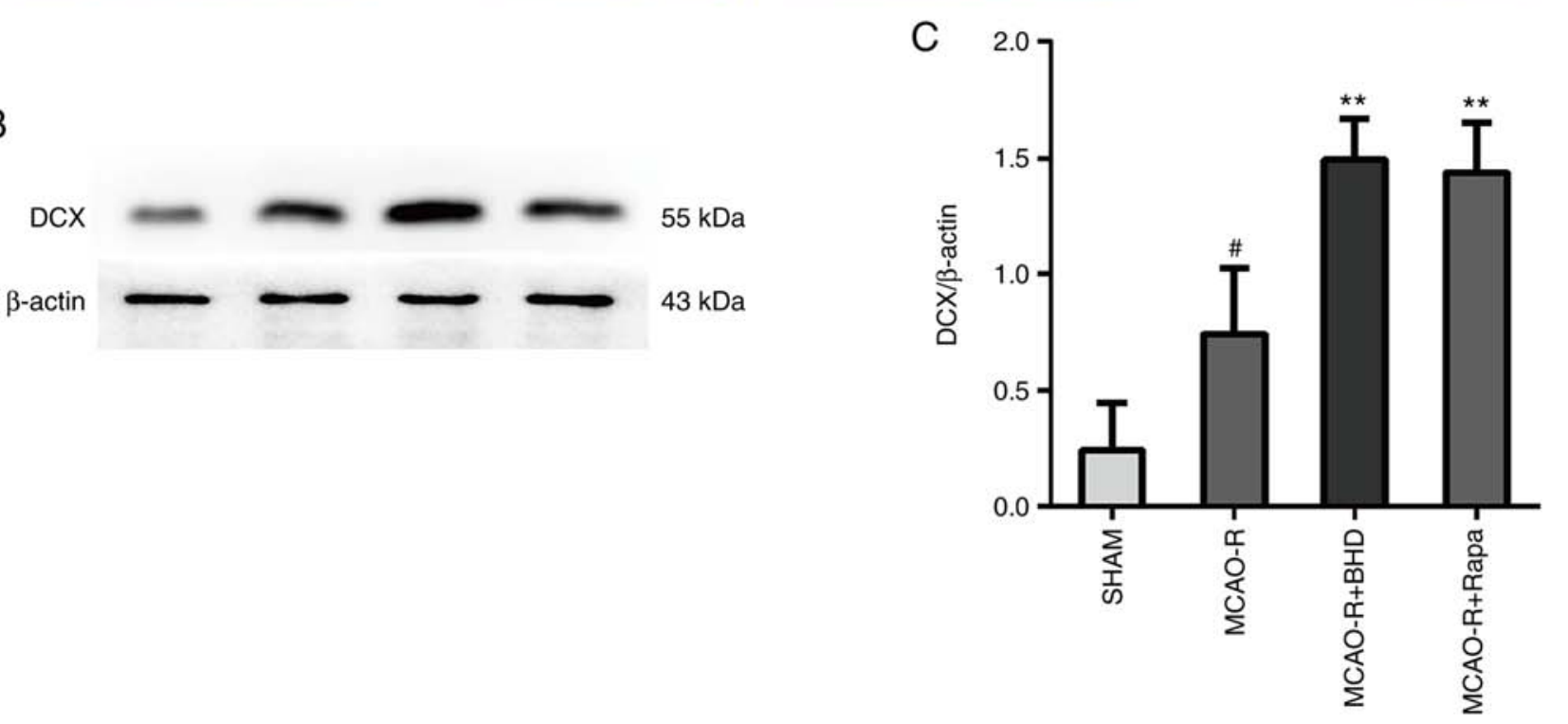

Figure 4. BHD promotes neurogenesis after MCAO-R. (A) Immunofluorescence analysis performed with DCX positive cells in the subgranular zone of the hippocampal dentate gyrus of rats (green), nuclei were stained with DAPI (blue). Scale bar=20 $\mu \mathrm{m}$. (B and C) Quantification of DCX by western blot. The data are expressed as means \pm standard deviation $(n=3)$. Statistical comparisons were performed with one-way ANOVA followed by Dunnett's t-test. ${ }^{\#} \mathrm{P}<0.05$ vs. sham group; ${ }^{* *} \mathrm{P}<0.01$ vs. MCAO-R group. BHD, Buyang Huanwu Decoction; MCAO-R, middle cerebral artery occlusion and reperfusion; DCX, doublecortin on the X-chromosome; Rapa, rapamycin.

dysfunction cannot clear the excessive ROS, thus triggering further pathological changes, such as calcium overload and excitotoxicity (53). There is a negative feedback regulation response between ROS and autophagy in mitochondria; mitochondria-produced ROS can activate autophagy and when autophagy is activated, ROS is eliminated (54). MDA is an important target that reflects the body's anti-oxidative potential and can indirectly reflect tissue peroxidation damage. CAT 

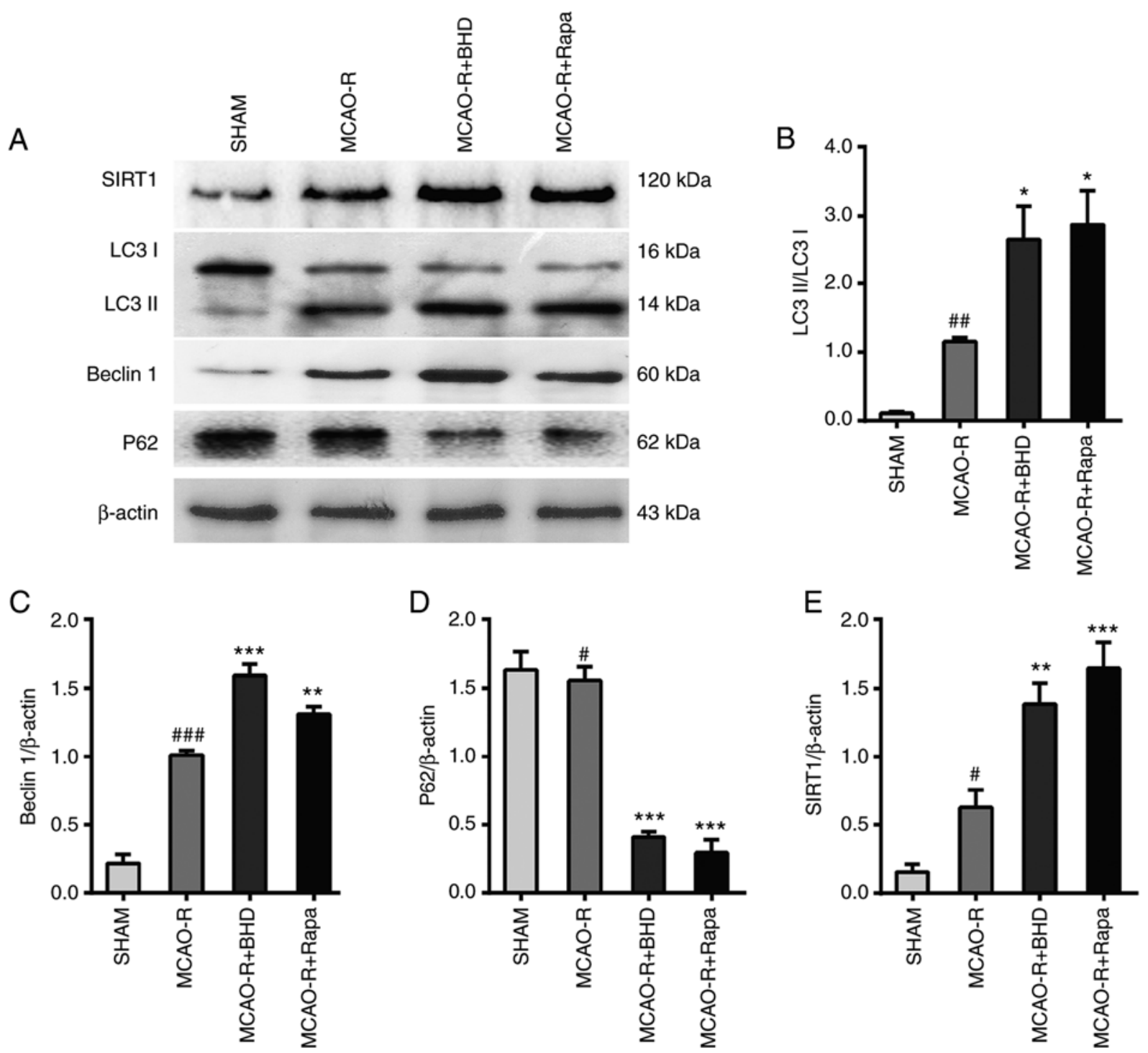

Figure 5. BHD activates SIRT1/autophagy of rat cerebral peri-ischemic area following MCAO-R. (A) Western blots of LC3, beclin1, P62, SIRT1 in BHD-treated MCAO-R rats. (B-E) Quantitative analysis of the immunoblotted proteins was performed with Image J. Statistical comparisons were carried out with ANOVA followed by Tukey's test. Data are presented as means \pm standard deviation $(n=3) .{ }^{~} \mathrm{P}<0.05$ vs. sham group; ${ }^{*} \mathrm{P}<0.05,{ }^{* *} \mathrm{P}<0.01,{ }^{* * *} \mathrm{P}<0.001$ vs $\mathrm{MCAO}-\mathrm{R}$ group. BHD, Buyang Huanwu Decoction; SIRT, sirtuin; MCAO-R, middle cerebral artery occlusion and reperfusion; Rapa, rapamycin.

and GSH-PX are two important peroxidases in the body. BHD can decrease the MDA level in the ischemic penumbra and increase the CAT and GSH-PX levels. Therefore, BHD can reduce oxidative stress damage following MCAO-R, which might be associated with the activation of the SIRT1/autophagy pathway.

Neurological deficits, such as hemiplegia and sensory disturbances, are the most common sequelae after stroke (55). Apoptosis is a process of programmed cell death, which is activated following cerebral ischemia injury and the production of ROS and inflammation during reperfusion (56). Autophagy and apoptosis both a form of cell self-regulation and the association between them is complex. Studies have shown that the inhibition of autophagy may lead to a shortage of bioenergy, thereby triggering cell apoptosis $(57,58)$. BDNF, a critical growth factor, has been shown to promote neuronal survival and regulate different neuronal functions (42). BHD significantly ameliorated neurological deficit and the level of brain damage in rats after MCAO-R. In the present study, following MCAO-R, extensive neuronal death was observed, which was accompanied by the disappearance of cytoplasmic bodies, swelling of cell bodies, nuclear condensation and sparse Nissl bodies, whereas sham group neurons exhibited clear and large cell nuclei and Nissl bodies. However, treatment with BHD reversed these changes. The immunofluorescence results demonstrated that MCAO-R significantly decreased the expression of BDNF, whereas post-surgical treatment with BHD could markedly increase it compared with the MCAO-R group. Thus, BHD could protect neurons against MCAO-R, which may have been associated with the activation of the SIRT1/autophagy pathway.

Neurogenesis is hypothesized to be restricted to embryonic development, ceasing after birth. However, adult neurogenesis has been detected to occur throughout the lifetime of various mammals (59). Adult neural stem cells in the subventricular zone of the lateral ventricle and the dentate gyrus of the hippocampus can be activated following stroke, to then proliferate and produce neuroblasts for the repair of damaged 

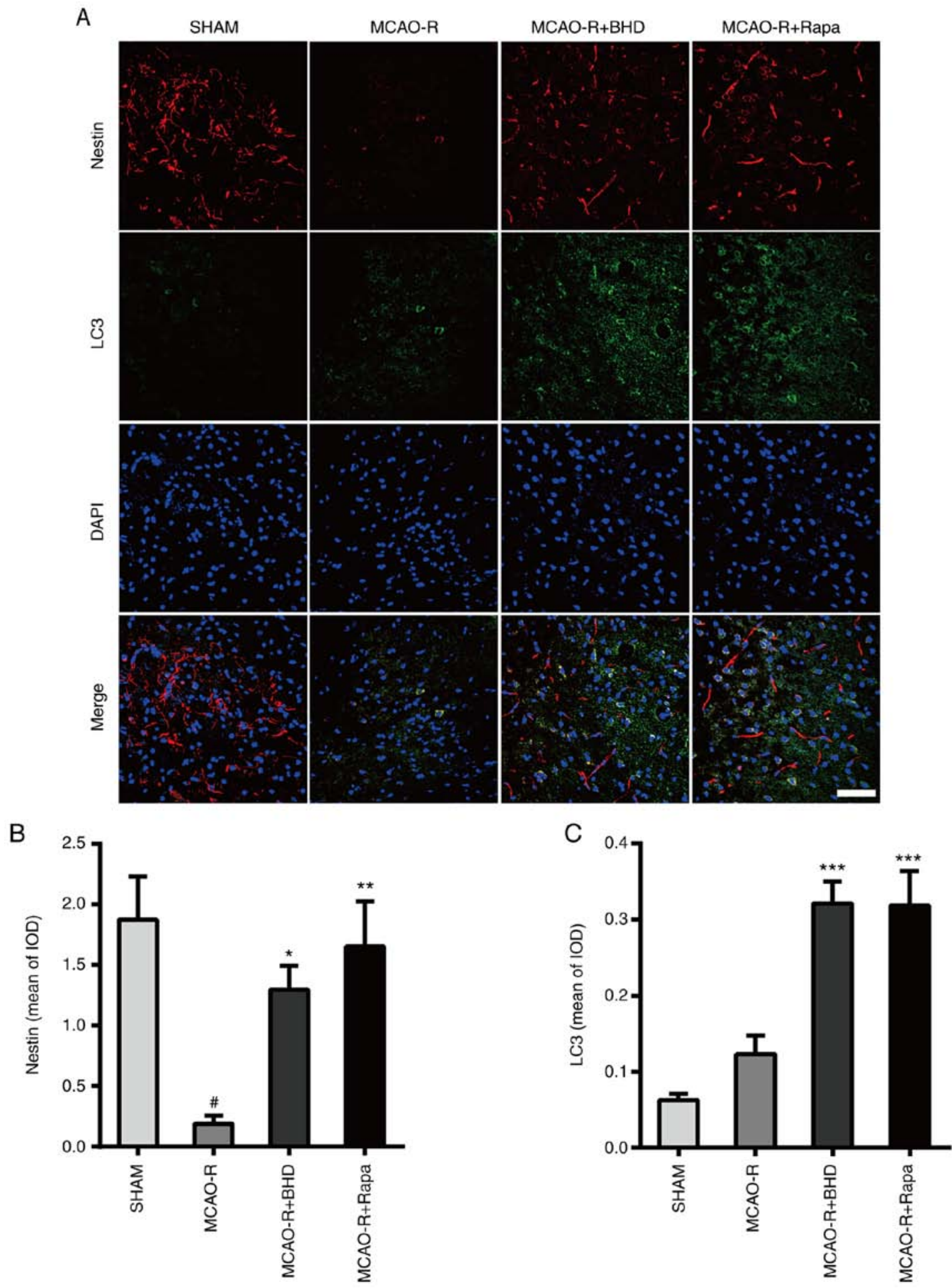

Figure 6. The BHD-induced neuroprotection is correlated with enhancement of autophagy. (A) The distribution of colocalization of nestin with the LC3 following MCAO-R in rat cerebral peri-ischemic area. Immunofluorescence images showing the colocalization of nestin (red) with LC3 (green). Nuclei were stained with DAPI (blue). Scale bar $=20 \mu \mathrm{m}$. The immunofluorescence intensity of (B) nestin and (C) LC 3 . The data are expressed as means \pm standard deviation $(\mathrm{n}=3)$. Statistical comparisons were performed with one-way ANOVA followed by Dunnett's post hoc test. ${ }^{\#} \mathrm{P}<0.05,{ }^{\# \#} \mathrm{P}<0.01$, ${ }^{\# \# \#} \mathrm{P}<0.001$ vs. sham group; ${ }^{*} \mathrm{P}<0.05,{ }^{* *} \mathrm{P}<0.01,{ }^{* * *} \mathrm{P}<0.001$ vs. MCAO-R group. BHD, Buyang Huanwu Decoction; SIRT, sirtuin; MCAO-R, middle cerebral artery occlusion and reperfusion; Rapa, rapamycin.

neurons $(60,61)$. A number of studies have investigated the role of autophagy in embryonic and adult neural stem cells. In the adult mammalian brain, the most studied neural stem cells, such as those located in the subventricular zone of the lateral ventricle and the subgranular zone of the hippocampal dentate gyrus, are located in a relatively hypoxic environment, which is a necessary condition for stem cells $(62,63)$. Through autophagy, a low level of ROS appears to be maintained, to ensure the slow circulation of neural stem cells (64). DCX is a microtubule and actin filament-associated protein (65). Due to its specific expression in neural precursors and newly generated immature neurons in several regions of the 
brain, DCX is used as a specific marker to assess potential neurogenesis in the adult brain $(66,67)$. Nestin is expressed in neural stem and progenitor cells, which may participate in neurogenesis following stroke $(18,68)$. Several studies have shown that BHD can induce neurogenesis during the occurrence of stroke $(30,69)$. In the present study, the expression of DCX in the MCAO-R + BHD group was increased compared with the MCAO-R group. These data demonstrated that the neuroprotective effect of BHD may be related to neurogenesis and autophagy. A double stain of a protein marker for neural stem cell (nestin) and autophagy-related protein (LC3) were performed to illustrate whether the neurogenesis of BHD is related to its regulation of autophagy. Confocal microscopy demonstrated that nestin and LC3 isoforms were located in living post-ischemic cells. These data provided evidence to suggest that BHD increased neurogenesis in the peri-ischemic area of rat brains on day 5 following MCAO-R, which might be associated with the activation of the SIRT1/autophagy pathway. SIRT1, a nicotinamide adenine dinucleotide-positive-dependent deacetylase, is a well-known modulator of aging (70). SIRT1 has been shown to exert anti-inflammatory, anti-apoptotic and anti-oxidative effects, promote DNA repair, maintain energy metabolism and regulate autophagy following the occurrence of stroke (71). SIRT1 can interact with several essential components of autophagy (22). Furthermore, research has shown that SIRT1 protects the brain during stroke, potentially through the activation of autophagy pathways (72). In the present study, BHD was proven to elevate SIRT1 expression, which may be an upstream autophagic protein.

In conclusion, the present study found that BHD may exert a neuroprotective effect and promote neurogenesis in MCAO-R rats by regulating the SIRT1/autophagy pathway in the peri-ischemic area of the brain. The findings of this study suggest that BHD may be a promising treatment for stroke, and that the SIRT1/autophagy pathway serves as a potential target for future therapies. However, the main limitation of this experiment is that the association between BHD and SIRT1/autophagy was not examined in-depth.

\section{Acknowledgements}

Not applicable.

\section{Funding}

The present study was supported by the National Natural Science Foundation of China (grant no. 81673717) and Guangdong Provincial Key Laboratory of Research on Emergency in TCM (grant no. 2017B030314176).

\section{Availability of data and materials}

The datasets used and/or analyzed during the current study are available from the corresponding author on reasonable request.

\section{Authors' contributions}

LG, QW and SJZ designed the experiments. HL, DP and YZ performed the experiments. HL wrote the manuscript. DP and SJZ modified the manuscript. HL and DP confirmed the authenticity of all the raw data. All authors reviewed and approved the final manuscript.

\section{Ethics approval and consent to participate}

All rat experiments and protocols were approved by the Experimental Animal Centre of Guangzhou University of Chinese Medicine of China (IACUC Approval no. 20190310001) on March 10, 2019.

\section{Patient consent for publication}

Not applicable.

\section{Competing interests}

The authors declare that they have no competing interests.

\section{References}

1. Benjamin EJ, Virani SS, Callaway CW, Chamberlain AM, Chang AR, Cheng S, Chiuve SE, Cushman M, Delling FN, Deo R, et al: Heart disease and stroke statistics-2018 update: A report from the American heart association. Circulation 137: e67-e492, 2018.

2. Zhou M, Wang H, Zhu J, Chen W, Wang L, Liu S, Li Y, Wang L, Liu Y, Yin P, et al: Cause-specific mortality for 240 causes in China during 1990-2013: A systematic subnational analysis for the Global Burden of Disease Study 2013. Lancet 387: 251-272, 2016.

3. GBD 2016 Lifetime Risk of Stroke Collaborators; Feigin VL, Nguyen G, Cercy K, Johnson CO, Alam T, Parmar PG, Abajobir AA, Abate KH, Abd-Allah F, et al: Global, regional, and country-specific lifetime risks of stroke, 1990 and 2016. N Engl J Med 379: 2429-2437, 2018.

4. Dai W, Liu X, Zhang Z, Chen J, Guo R, Zheng H, Jin X, Wen S, Gao Y, Li T, et al: A two-level model for the analysis of syndrome of acute ischemic stroke: From diagnostic model to molecular mechanism. Evid Based Complement Alternat Med 2013: 293010, 2013

5. Han CH, Kim M, Cho SY, Jung WS, Moon SK, Park JM, Ko CN, Cho KH and Kwon S: Adjunctive herbal medicine treatment for patients with acute ischemic stroke: A systematic review and meta-analysis. Complement Ther Clin Pract 33: 124-137, 2018.

6. Hodges $\mathrm{H}$ : Graft-induced recovery of cognitive function after diffuse and focal brain damage: Implications for neural transplantation in man. Zh Vyssh Nerv Deiat Im I P Pavlova 45: 29-58, 1995.

7. Hossmann KA: Pathophysiology and therapy of experimental stroke. Cell Mol Neurobiol 26: 1057-1083, 2006.

8. Patel RAG and McMullen PW: Neuroprotection in the treatment of acute ischemic stroke. Prog Cardiovasc Dis 59: 542-548, 2017.

9. George PM and Steinberg GK: Novel stroke therapeutics: Unraveling stroke pathophysiology and its impact on clinical treatments. Neuron 87: 297-309, 2015.

10. Prabhakaran S, Ruff I and Bernstein RA: Acute stroke intervention: A systematic review. JAMA 313: 1451-1462, 2015.

11. Zhang S, Boyd J, Delaney K and Murphy TH: Rapid reversible changes in dendritic spine structure in vivo gated by the degree of ischemia. J Neurosci 25: 5333-5338, 2005.

12. Nakagiri A, Sunamoto M and Murakami M: NADPH oxidase is involved in ischaemia/reperfusion-induced damage in rat gastric mucosa via ROS production-role of NADPH oxidase in rat stomachs. Inflammopharmacology 15: 278-281, 2007.

13. Gage FH: Adult neurogenesis in mammals. Science 364: 827-828, 2019.

14. Cavallucci V, Fidaleo M and Pani G: Nutrients and neurogenesis: The emerging role of autophagy and gut microbiota. Curr Opin Pharmacol 50: 46-52, 2020.

15. Duan X, Chen B, Cui Y, Zhou L, Wu C, Yang Z, Wen Y, Miao X, $\mathrm{Li} \mathrm{Q}$, Xiong L and He J: Ready player one? Autophagy shapes resistance to photodynamic therapy in cancers. Apoptosis 23: 587-606, 2018. 
16. Liang K, Zhu L, Tan J, Shi W, He Q and Yu B: Identification of autophagy signaling network that contributes to stroke in the ischemic rodent brain via gene expression. Neurosci Bull 31: 480-490, 2015

17. Sheng R, Zhang LS, Han R, Liu XQ, Gao B and Qin ZH: Autophagy activation is associated with neuroprotection in a rat model of focal cerebral ischemic preconditioning. Autophagy 6: 482-494, 2010.

18. Su J, Zhang T, Wang K, Zhu T and Li X: Autophagy activation contributes to the neuroprotection of remote ischemic perconditioning against focal cerebral ischemia in rats. Neurochem Res 39: 2068-2077, 2014.

19. Gao L, Jiang T, Guo J, Liu Y, Cui G, Gu L, Su L and Zhang Y: Inhibition of autophagy contributes to ischemic postconditioning-induced neuroprotection against focal cerebral ischemia in rats. PLoS One 7: e46092, 2012.

20. Vázquez P, Arroba AI, Cecconi F, de la Rosa EJ, Boya P and de Pablo F: Atg5 and Ambral differentially modulate neurogenesis in neural stem cells. Autophagy 8: 187-199, 2012.

21. Lv X, Jiang H, Li B, Liang Q, Wang S, Zhao Q and Jiao J: The crucial role of Atg 5 in cortical neurogenesis during early brain development. Sci Rep 4: 6010, 2014

22. Chen X, Pan Z, Fang Z, Lin W, Wu S, Yang F, Li Y, Fu H, Gao $\mathrm{H}$ and Li S: Omega-3 polyunsaturated fatty acid attenuates traumatic brain injury-induced neuronal apoptosis by inducing autophagy through the upregulation of SIRT1-mediated deacetylation of Beclin-1. J Neuroinflammation 15: 310, 2018

23. Tang Q, Len Q, Liu Z and Wang W: Overexpression of miR-22 attenuates oxidative stress injury in diabetic cardiomyopathy via Sirt 1. Cardiovase Ther 36: 2018

24. Carloni S, Albertini MC, Galluzzi L, Buonocore G, Proietti F and Balduini W: Melatonin reduces endoplasmic reticulum stress and preserves sirtuin 1 expression in neuronal cells of newborn rats after hypoxia-ischemia. J Pineal Res 57: 192-199, 2014.

25. Wang Q, Liu M, Liu WW, Hao WB, Tashiro S, Onodera S and Ikejima T: In vivo recovery effect of silibinin treatment on streptozotocin-induced diabetic mice is associated with the modulations of Sirt-1 expression and autophagy in pancreatic $\beta$-cell. J Asian Nat Prod Res 14: 413-423, 2012

26. Guo Q, Zhong M, Xu H, Mao X, Zhang Y and Lin N: A systems biology perspective on the molecular mechanisms underlying the therapeutic effects of buyang huanwu decoction on ischemic stroke. Rejuvenation Res 18: 313-325, 2015

27. Hao CZ, Wu F, Shen J, Lu L, Fu DL, Liao WJ and Zheng GQ Clinical efficacy and safety of buyang huanwu decoction for acute ischemic stroke: A systematic review and meta-analysis of 19 randomized controlled trials. Evid Based Complement Alternat Med 2012: 630124, 2012.

28. Zheng XW, Shan CS, Xu QQ, Wang Y, Shi YH, Wang Y and Zheng GQ: Buyang huanwu decoction targets SIRT1/VEGF pathway to promote angiogenesis after cerebral ischemia/reperfusion injury. Front Neurosci 12: 911, 2018

29. Liu B, Cai G, Yi J and Chen X: Buyang huanwu decoction regulates neural stem cell behavior in ischemic brain. Neural Regen Res 8: 2336-2342, 2013 .

30. Luo L, Deng S, Yi J, Zhou S, She Y and Liu B: Buyang huanwu decoction ameliorates poststroke depression via promoting neurotrophic pathway mediated neuroprotection and neurogenesis. Evid Based Complement Alternat Med 2017: 4072658, 2017.

31. Wang Y, Liu X, Hu T, Li X, Chen Y, Xiao G, Huang J, Chang Y, Zhu Y, Zhang $\mathrm{H}$ and Wang Y: Astragalus saponins improves stroke by promoting the proliferation of neural stem cells through phosphorylation of Akt. J Ethnopharmacol 277: 114224, 2021.

32. Li JH, Liu AJ, Li HQ, Wang Y, Shang HC and Zheng GQ Buyang huanwu decoction for healthcare: Evidence-based theoretical interpretations of treating different diseases with the same method and target of vascularity. Evid Based Complement Alternat Med 2014: 506783, 2014.

33. Zhang ZQ, Song JY, Jia YQ and Zhang YK: Buyanghuanwu decoction promotes angiogenesis after cerebral ischemia/reperfusion injury: Mechanisms of brain tissue repair. Neural Regen Res 11: 435-440, 2016.

34. Zhang WW, Xu F, Wang D, Ye J and Cai SQ: Buyang huanwu decoction ameliorates ischemic stroke by modulating multiple targets with multiple components: In vitro evidences. Chin J Nat Med 16: 194-202, 2018

35. Luo C, Ouyang MW, Fang YY, Li SJ, Zhou Q, Fan J, Qin ZS and Tao T: Dexmedetomidine protects mouse brain from ischemia-reperfusion injury via inhibiting neuronal autophagy through Up-Regulating HIF-1 $\alpha$. Front Cell Neurosci 11: 197, 2017.
36. Shen J, Zhu Y, Huang K, Jiang H, Shi C, Xiong X, Zhan R and Pan J: Buyang Huanwu Decoction attenuates $\mathrm{H} 2 \mathrm{O} 2$-induced apoptosis by inhibiting reactive oxygen species-mediated mitochondrial dysfunction pathway in human umbilical vein endothelial cells. BMC Complement Altern Med 16: 154, 2016.

37. Huang YT, Chen YY, Lai YH, Cheng CC, Lin TC, Su YS, Liu CH and Lai PC: Resveratrol alleviates the cytotoxicity induced by the radiocontrast agent, ioxitalamate, by reducing the production of reactive oxygen species in HK-2 human renal proximal tubule epithelial cells in vitro. Int J Mol Med 37: 83-91, 2016.

38. Chong ZZ, Shang YC, Zhang L, Wang S and Maiese K: Mammalian target of rapamycin: Hitting the bull's-eye for neurological disorders. Oxid Med Cell Longev 3: 374-391, 2010.

39. Xia D, Zhang Z and Zhao Y: Acteoside attenuates oxidative stress and neuronal apoptosis in rats with focal cerebral ischemia-reperfusion injury. Biol Pharm Bull 41: 1645-1651, 2018.

40. Yang J, Yan H, Li S and Zhang M: Berberine ameliorates MCAO induced Cerebral Ischemia/Reperfusion injury via activation of the BDNF-TrkB-PI3K/Akt signaling pathway. Neurochem Res 43: 702-710, 2018.

41. Li YQ, Hui ZR, Tao T, Shao KY, Liu Z, Li M and Gu LL: Protective effect of hypoxia inducible factor-1 $\alpha$ gene therapy using recombinant adenovirus in cerebral ischaemia-reperfusion injuries in rats. Pharm Biol 58: 438-446, 2020.

42. Song M, Mohamad O, Gu X, Wei L and Yu SP: Restoration of intracortical and thalamocortical circuits after transplantation of bone marrow mesenchymal stem cells into the ischemic brain of mice. Cell Transplant 22: 2001-2015, 2013.

43. Lee JH, Yu WH, Kumar A, Lee S, Mohan PS, Peterhoff CM, Wolfe DM, Martinez-Vicente M, Massey AC, Sovak G, et al: Lysosomal proteolysis and autophagy require presenilin 1 and are disrupted by Alzheimer-related PS1 mutations. Cell 141: $1146-1158,2010$.

44. Hou K, Xu D, Li F, Chen S and Li Y: The progress of neuronal autophagy in cerebral ischemia stroke: Mechanisms, roles and research methods. J Neurol Sci 400: 72-82, 2019.

45. Carloni S, Girelli S, Scopa C, Buonocore G, Longini M and Balduini W: Activation of autophagy and Akt/CREB signaling play an equivalent role in the neuroprotective effect of rapamycin in neonatal hypoxia-ischemia. Autophagy 6: 366-377, 2010

46. Zhang Y, Cao Y and Liu C: Autophagy and ischemic stroke. Adv Exp Med Biol 1207: 111-134, 2020.

47. Kim KJ and Namgung U: Facilitating effects of Buyang Huanwu decoction on axonal regeneration after peripheral nerve transection. J Ethnopharmacol 213: 56-64, 2018.

48. Zhang M, Chai Y, Liu T, Xu N and Yang C: Synergistic effects of Buyang Huanwu decoction and embryonic neural stem cell transplantation on the recovery of neurological function in a rat model of spinal cord injury. Exp Ther Med 9: 1141-1148, 2015.

49. Min L, Ling W, Hua R, Qi H, Chen S, Wang H, Tang L and Shangguan W: Anti-angiogenic therapy for normalization of tumor vasculature: A potential effect of Buyang Huanwu decoction on nude mice bearing human hepatocellular carcinoma xenografts with high metastatic potential. Mol Med Rep 13: 2518-2526, 2016

50. Wu L, Zhang W, Li H, Chen BY, Zhang GM, Tang YH, He FY and Deng CQ: Inhibition of aortic intimal hyperplasia and cell cycle protein and extracellular matrix protein expressions by BuYang HuanWu Decoction. J Ethnopharmacol 125: 423-435, 2009.

51. Rodrigo R, Fernández-Gajardo R, Gutiérrez R, Matamala JM, Carrasco R, Miranda-Merchak A and Feuerhake W: Oxidative stress and pathophysiology of ischemic stroke: Novel therapeutic opportunities. CNS Neurol Disord Drug Targets 12: 698-714, 2013.

52. Orellana-Urzúa S, Rojas I, Líbano L and Rodrigo R: Pathophysiology of ischemic stroke: Role of oxidative stress. Curr Pharm Des 26: 4246-4260, 2020.

53. Wu MY, Yiang GT, Liao WT, Tsai AP, Cheng YL, Cheng PW, $\mathrm{Li} \mathrm{CY}$ and $\mathrm{Li} \mathrm{CJ}$ : Current mechanistic concepts in ischemia and reperfusion injury. Cell Physiol Biochem 46: 1650-1667, 2018.

54. Filomeni G, De Zio D and Cecconi F: Oxidative stress and autophagy: The clash between damage and metabolic needs. Cell Death Differ 22: 377-388, 2015.

55. Yamashita $\mathrm{T}$ and Abe K: Recent progress in therapeutic strategies for ischemic stroke. Cell Transplant 25: 893-898, 2016. 
56. Kalogeris T, Bao Y and Korthuis RJ: Mitochondrial reactive oxygen species: A double edged sword in ischemia/reperfusion vs preconditioning. Redox Biol 2: 702-714, 2014.

57. Lum JJ, DeBerardinis RJ and Thompson CB: Autophagy in metazoans: Cell survival in the land of plenty. Nat Rev Mol Cel Biol 6: 439-448, 2005.

58. Kroemer $\mathrm{G}$ and Jäättelä $\mathrm{M}$ : Lysosomes and autophagy in cell death control. Nat Rev Cancer 5: 886-897, 2005.

59. Eriksson PS, Perfilieva E, Björk-Eriksson T, Alborn AM, Nordborg C, Peterson DA and Gage FH: Neurogenesis in the adult human hippocampus. Nat Med 4: 1313-1317, 1998.

60. Zhang RL, Zhang ZG, Zhang L and Chopp M: Proliferation and differentiation of progenitor cells in the cortex and the subventricular zone in the adult rat after focal cerebral ischemia. Neuroscience 105: 33-41, 2001.

61. Jin K, Minami M, Lan JQ, Mao XO, Batteur S, Simon RP and Greenberg DA: Neurogenesis in dentate subgranular zone and rostral subventricular zone after focal cerebral ischemia in the rat. Proc Natl Acad Sci USA 98: 4710-4715, 2001.

62. Doetsch F, Caillé I, Lim DA, García-Verdugo JM and Alvarez-Buylla A: Subventricular zone astrocytes are neural stem cells in the adult mammalian brain. Cell 97: 703-716, 1999.

63. Palmer TD, Takahashi J and Gage FH: The adult rat hippocampus contains primordial neural stem cells. Mol Cell Neurosci 8: 389-404, 1997.

64. Boya P, Codogno P and Rodriguez-Muela N: Autophagy in stem cells: Repair, remodelling and metabolic reprogramming. Development 145: dev146506, 2018.

65. Dhaliwal J, Xi Y, Bruel-Jungerman E, Germain J, Francis F and Lagace DC: Doublecortin (DCX) is not essential for survival and differentiation of Newborn Neurons in the adult mouse dentate gyrus. Front Neurosci 9: 494, 2015.

66. Fasemore TM, Patzke N, Kaswera-Kyamakya C, Gilissen E, Manger PR and Ihunwo AO: The Distribution of Ki-67 and doublecortin-immunopositive cells in the brains of three strepsirrhine primates: Galago demidoff, perodicticus potto, and Lemur catta. Neuroscience 372: 46-57, 2018.
67. Shahsavani M, Pronk RJ, Falk R, Lam M, Moslem M, Linker SB, Salma J, Day K, Schuster J, Anderlid BM, et al: An in vitro model of lissencephaly: Expanding the role of DCX during neurogenesis. Mol Psychiatry 23: 1674-1684, 2018.

68. Bojnordi MN, Azizi H, Skutella T, Movahedin M, Pourabdolhossein F, Shojaei A and Hamidabadi HG: Differentiation of spermatogonia stem cells into functional mature neurons characterized with differential gene expression. Mol Neurobiol 54: 5676-5682, 2017.

69. Chen X, Chen H, He Y, Fu S, Liu H, Wang Q and Shen J: Proteomics-guided study on buyang huanwu decoction for its neuroprotective and neurogenic mechanisms for transient ischemic stroke: Involvements of EGFR/PI3K/Akt/Bad/14-3-3 and Jak2/Stat3/Cyclin D1 signaling cascades. Mol Neurobiol 57: 4305-4321, 2020.

70. Lapierre LR, Kumsta C, Sandri M, Ballabio A and Hansen M: Transcriptional and epigenetic regulation of autophagy in aging. Autophagy 11: 867-880, 2015.

71. Zhang JF, Zhang YL and Wu YC: The role of sirt1 in ischemic stroke: Pathogenesis and therapeutic strategies. Front Neurosci 12: 833, 2018

72. Wang P, Guan YF, Du H, Zhai QW, Su DF and Miao CY: Induction of autophagy contributes to the neuroprotection of nicotinamide phosphoribosyltransferase in cerebral ischemia. Autophagy 8: 77-87, 2012.

This work is licensed under a Creative Commons Attribution-NonCommercial-NoDerivatives 4.0 International (CC BY-NC-ND 4.0) License. 